SHORT REPORT

\title{
Alterations of muscarinic acetylcholine receptors in atypical Pick's disease without Pick bodies
}

\author{
T Odawara, K Shiozaki, E Iseki, H Hino, K Kosaka
}

J Neurol Neurosurg Psychiatry 2003;74:965-967

Background: Atypical Pick's disease without Pick bodies is a type of frontotemporal dementia characterised by semantic dementia and temporal dominant lobar atrophy with ubiquitinopathy. No neurochemical analyses have ever been reported in this condition.

Objective: To investigate muscarinic acetylcholine receptors (mAchR) and their subtypes (MI-M4) in atypical Pick's disease.

Subjects: Five cases of atypical Pick's disease were studied. They were compared with nine control cases, 11 cases of Alzheimer's disease, and seven cases of dementia with Lewy bodies.

Methods: A $\left[{ }^{3} \mathrm{H}\right]$ quinuclidinyl benzilate (QNB) binding assay and an immunoprecipitation assay using subtype specific antisera were used.

Results: The total amount of $m A$ chR in the temporal cortex was lower in atypical Pick's disease than in controls or Alzheimer's disease cases, but there were no significant differences between the three groups in the frontal cortex. In the temporal cortex, there was a smaller proportion of M1 receptors in atypical Pick's disease than in the controls or in the patients with Alzheimer's disease and dementia with Lewy bodies. In contrast, the proportion of $M 2$ receptor was higher in atypical Pick's disease than in the other three groups.

Conclusions: Depletion of postsynaptic cholinoreceptive neurones in the temporal cortex is more severe in atypical Pick's disease than in other neurodegenerative dementing disorders.

$\mathrm{P}$ ick's disease is a rare form of neurodegenerative dementia, neuropathologically characterised by circumscribed frontotemporal lobe atrophy as well as by severe neuronal loss and gliosis in the atrophied regions. ${ }^{12}$ Nowadays, the use of the term of "Pick's disease" is restricted to cases showing lobar atrophy with Pick bodies and tauopathy, and Pick's disease is included under the nomenclature of frontotemporal dementia. ${ }^{3}$ In contrast, cases showing lobar atrophy without Pick bodies are neuropathologically heterogeneous. Some of these cases have clinical features of semantic dementia, which is among the clinical diagnostic criteria of frontotemporal lobar degeneration (FTLD), ${ }^{4}$ and show temporal dominant lobar atrophy-hence the name "atypical Pick's disease ${ }^{\prime 5} .{ }^{5}$ Although atypical Pick's disease shares ubiquitinopathy with the dementia that occurs with motor neurone disease, ${ }^{57}$ it is differentiated from that disorder by absence of clinical features of amyotrophic lateral sclerosis and preservation of lower motor neurones. ${ }^{6}$ Atypical Pick's disease is considered to be a temporal dominant type of fronto-temporal dementia.

Neocortical cholinergic deficits have been extensively investigated in some forms of neurodegenerative dementia in relation to memory disturbance, especially in Alzheimer's dis-

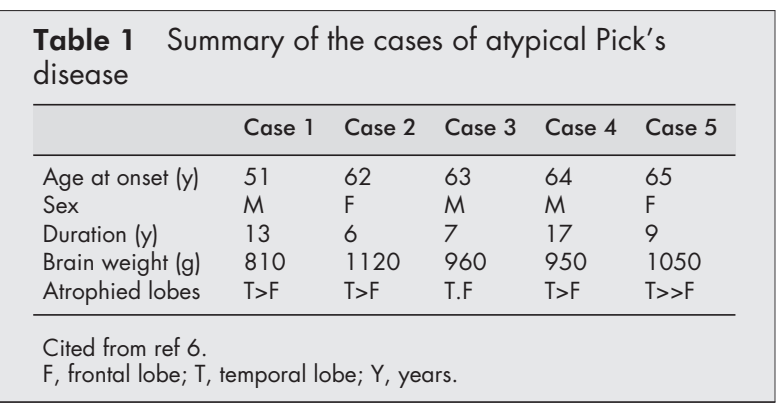

ease and dementia with Lewy bodies. ${ }^{8-10}$ However, investigation into the cholinergic systems in fronto-temporal dementia has been inadequate, and have only been undertaken in the frontal lobe degeneration type of dementia and in the Pick type with Pick bodies. ${ }^{11}{ }^{12}$ Systemic studies of muscarinic acetylcholine receptors (mAchR) in atypical Pick's disease have never been done.

In this study, we estimated the total amounts of mAchR and the levels of the mAchR subtypes in atypical Pick's disease.

\section{METHODS}

We examined brains from five cases of atypical Pick's disease (mean (SD) age, 71.4 (6.4) years, range 64 to 81 ; mean postmortem delay to specimen retrieval 3.4 (1.4) hours (table 1). The clinical, neuropathological, and neurochemical features of these cases have been described in detail previously. ${ }^{6}$ In summary, all cases showed semantic dementia and had a clinical diagnosis of FTLD. There was no family history of this disorder in any case. Neuropathologically, all cases showed lobar atrophy of the frontal and temporal lobes with temporal lobe dominance. There was moderate to severe degeneration with neuronal loss and gliosis in the affected cerebral cortices and subcortical nuclei. In the pyramidal tract, the right cerebral peduncle was degenerated but the hypoglossal nucleus and the anterior horn of the spinal cord were preserved.

Immunohistochemical analyses in all cases showed ubiquitinopathy with ubiquitin accumulation in intraneuronal and dendritic inclusions in the atrophied cortex. Immunoblots of samples from the cases did not show detectable amounts of insoluble tau.

The dissected brain tissues were immediately frozen in liquid nitrogen, and then stored at $-80^{\circ} \mathrm{C}$ until used.

For comparison, we studied 11 cases of Alzheimer's disease (mean age 78.4 (8.7) years, range 63 to 86; mean postmortem delay 2.6 (1.9) hours), seven cases of dementia with Lewy

Abbreviations: ChAT, choline acetyltransferase; FTLD, frontotemporal lobar degeneration; $m A c h R$, muscarinic acetylcholine receptors; QNB, $\left[{ }^{3} \mathrm{H}\right]$ quinuclidinyl benzilate 
Table 2 Immunoprecipitation of muscarinic acetylcholine receptor subtypes using subtype specific antisera

\begin{tabular}{|c|c|c|c|c|c|}
\hline \multirow[b]{2}{*}{ Disease } & \multirow{2}{*}{$\begin{array}{l}{\left[{ }^{3} \mathrm{H}\right] \text { QNB bound }} \\
(\mathrm{fmol} / \mathrm{mg})\end{array}$} & \multicolumn{4}{|c|}{ Proportions of precipitated mAchR (\%) } \\
\hline & & M1 & M2 & M3 & M4 \\
\hline \multicolumn{6}{|l|}{ Frontal cortex } \\
\hline Controls $(n=9)$ & $1440(414)$ & 50.6 & 9.6 & 6.5 & 10.0 \\
\hline DLB $(n=6)$ & $1128(262)$ & 50.5 & 8.4 & $9.8^{*}$ & 9.2 \\
\hline$A D(n=11)$ & 1407 (181) & 51.0 & 9.4 & $9.6^{* *}$ & 9.4 \\
\hline $\operatorname{aPD}(n=5)$ & 1076 (157) & 51.4 & 11.0 & $6.6 \dagger$ & 8.4 \\
\hline \multicolumn{6}{|c|}{ Temporal cortex } \\
\hline Controls $(n=9)$ & $1957(210)$ & 53.3 & 9.0 & 7.3 & 10.4 \\
\hline $\mathrm{DLB}(n=7)$ & $1120(95)^{* * *}$ & 58.1 & 8.5 & 8.2 & $6.8^{* *}$ \\
\hline$A D(n=11)$ & $1371(298)^{* * *}$ & 52.1 & $11.6 * \ddagger$ & 9.3 & 7.6 ** \\
\hline $\mathrm{aPD}(\mathrm{n}=5)$ & $797(194)^{* * *}+\dagger \dagger$ & 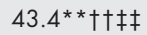 & 18.0***††‡‡ & 6.7 & 8.8 \\
\hline
\end{tabular}

Data are mean (SD) data from a representative experiment. The density of $m A c h R$ was estimated as specific binding with $\left[{ }^{3} \mathrm{H}\right] \mathrm{QNB}$, and ranged from 800 to $2500 \mathrm{fmol} / \mathrm{mg}$ membrane protein. The proportions of specially precipitated $m A$ chR subtypes were estimated as the differences in the amounts of bound $\left[{ }^{3} H\right] Q N B$ precipitated with specific antiserum and those precipitated with non-immune serum.

${ }^{*} p<0.05,{ }^{* *} p<0.01 ;{ }^{* * *} p<0.001 \vee$ controls; $t p<0.05,+\dagger p<0.01$, $+\dagger p<0.0015 \vee A D ; \neq p<0.01$, $\neq \neq p<0.001 \vee D L B$.

$A D$, Alzheimer's disease; aPD, atypical Pick's disease; DLB, dementia with Lewy bodies; mAchR, muscarinic acetylcholine receptors; QNB, $\left[{ }^{3} \mathrm{H}\right]$ quinuclidinyl benzilate.

bodies (mean age 77.9 (6.9) years, range 68 to 86 ; mean postmortem delay 4.4 (1.5) hours), and nine controls with no notable neuropathological findings (mean age 84.9 (5.3) years, range 75 to 91; mean postmortem delay 5.7 (5.6) hours). All cases selected for comparative study, except for two of the cases in the control group, had been used previously in another study. ${ }^{10}$

The methods used have been described previously in detail. ${ }^{10}$ In brief, the amounts of total mAchR in all cases were estimated at the specific binding sites of $\left[{ }^{3} \mathrm{H}\right]$ quinuclidinyl benzilate $(\mathrm{QNB})$ in membrane preparations from the frontal and temporal cortices. The samples of frontal and temporal cortices were prepared, respectively, from the frontal pole and the rostral parts of the temporal lobes that were most severely affected. The amounts of proteins were measured using an assay kit from Bio-Rad (Hercules, California, USA). An immunoprecipitation assay using specific antisera against AchR Ml, M2, M3, and M4 was done to estimate the levels of mAchR subtypes. Diverse antisera were raised against GST fusion proteins incorporating the putative third intracellular loop.

\section{Statistics}

One way analysis of variance followed by the least significant difference test was used to compare the values of total QNB binding and the proportions of subtypes Ml to M4. Statistical evaluation was done using the SPSS package.

\section{RESULTS}

The amounts of mAchR at the $\left[{ }^{3} \mathrm{H}\right] \mathrm{QNB}$ binding sites in membrane preparations ranged from 0.8 to $2.5 \mathrm{pmol} / \mathrm{mg}$ protein (frontal cortex) and from 0.8 to $2.1 \mathrm{pmol} / \mathrm{mg}$ protein (temporal cortex). In the temporal cortex, values for the $\left[{ }^{3} \mathrm{H}\right] \mathrm{QNB}$ binding sites in atypical Pick's disease were significantly lower than in the control or Alzheimer's disease groups, although no substantial differences were not found between atypical Pick's disease and dementia with Lewy bodies. In contrast, there were no significant differences in the binding sites in the frontal cortex among the various groups.

Membrane preparations from all cases were used for precipitation with each of the antisera against M1, M2, M3, and M4 receptors. The proportions of [3H] QNB-receptor complex precipitated with each of the antisera as solubilised complexes were estimated (table 2). In the frontal cortex, the proportion of $\left[{ }^{3} \mathrm{H}\right] \mathrm{QNB}-$ receptor complex precipitated with anti-M3 antiserum was significantly lower in atypical Pick's disease than in Alzheimer's disease. In the temporal cortex, the proportion of anti-Ml complex was significantly lower in atypical Pick's disease than in the other groups. In contrast, the proportion of anti-M2 complex was higher in atypical Pick's disease than in the other groups. Comparisons between the other three groups are omitted here, as they have been dealt with elsewhere. ${ }^{10}$

\section{DISCUSSION}

There have been few systematic neurochemical studies on cholinergic alterations in cases of fronto-temporal dementia. Wood et al reported that the activity of choline acetyltransferase (ChAT) and the numbers of mAchR were unchanged in the cerebral cortex in three cases of Pick's disease with Pick bodies. ${ }^{11}$ Both Hansen et al and Francis et al found that ChAT activity was similar to control values, and that the numbers of mAchR were decreased in the temporal cortex in all of seven cases of Pick's disease with Pick bodies, although the decrease was less than in Alzheimer's disease. ${ }^{12}{ }^{13}$ Procter et al showed that the numbers of mAchR did not fall in 10 cases of Pick's disease with Pick bodies or in six cases of fronto-temporal dementia of the frontal lobe degeneration type, but did fall in nine cases of Alzheimer's disease in the frontal, temporal, and parietal cortices. ${ }^{14}$ In summary, previous studies on Pick's disease with Pick bodies and the frontal lobe degeneration type of fronto-temporal dementia have shown that numbers of mAchR are either preserved or reduced to some extent, although ChAT activity-which indicates presynaptic cholinergic innervation-is preserved.

The incidence of atypical Pick's disease is similar to that of Pick's disease with Pick bodies in Japan. ${ }^{15}$ Our results show that the decrease in mAchR was more severe in the temporal cortex in atypical Pick's disease than in controls or Alzheimer's disease cases. However, no substantial differences were found in the total amounts of mAchR between atypical Pick's disease and dementia with Lewy bodies. This may accounted for the finding that the neocortical cholinergic deficit is more extensive in dementia with Lewy bodies than in Alzheimer's disease. ${ }^{89}$

In the frontal cortex, the proportion of M3 receptors was lower in atypical Pick's disease than in Alzheimer's disease. This result may reflect the relatively higher proportion of M2 receptors in atypical Pick's disease, but this is not known for certain. In the temporal cortex, there was a decrease in the proportion of $\mathrm{Ml}$ receptors, the most abundant muscarinic acetylcholine receptor in the cerebral cortex, ${ }^{16}$ while the proportion of M2 receptors, which exist at presynapses of the 
cholinergic system, ${ }^{17}$ increased. The lower level of $\mathrm{Ml}$ receptors in atypical Pick's disease may reflect the greater severity of neuronal losses in the temporal cortex in this condition in comparison with Alzheimer's disease or dementia with Lewy bodies, because all cases of atypical Pick's disease showed severe neuronal loss with gliosis in the anterior portions of the temporal lobes. ${ }^{6}$ Moreover, the higher level of M2 in atypical Pick's disease raises the possibility that the presynaptic M2 receptors are upregulated as a result of the loss of cholinoreceptive neurones in the temporal cortex, although it is not possible to determine the absolute proportion of Ml to M4 receptors by our immunoprecipitation method. These findings suggest that projecting cholinergic neurones are relatively preserved, in contrast to the loss of cholinoreceptive neurones in the temporal cortex, although ChAT was not estimated in this study. A previous neuropathological study showed that the nucleus basalis of Meynert, from which the cholinergic neurones project to the temporal cortex, was mildly degenerated in Pick's disease, including atypical Pick's disease, ${ }^{18}$ supporting our hypothesis.

Acetylcholine esterase inhibitors are used in the treatment of Alzheimer's disease, and are also of potential use in dementia with Lewy bodies ${ }^{10}$ and vascular dementia. Unfortunately, our study suggests that these agents may be ineffective in treating cholinergic deficiency in patients suffering from atypical Pick's disease, because the cholinoreceptive neurones appear to be more severely affected than the projecting presynaptic cholinergic neurones.

\section{ACKNOWLEDGEMENTS}

This study was supported in part by grants-in-aid for scientific research from the Japan Ministry of Education, Science, Sports and Culture.

\section{Authors' affiliations}

T Odawara, E Iseki, H Hino, K Kosaka, Department of Psychiatry,

Yokohama City University School of Medicine, Yokohama, Japan

K Shiozaki, Yokohama Maioka Hospital, Yokohama

Competing interests: none declared

Correspondence to: Dr Toshinari Odawara, Department of Psychiatry, Yokohama City University School of Medicine, 3-9 Fukuura,

Kanazawa-ku, Yokohama 236-0004, Japan;

odaw1913@med.yokohama-cu.ac.jp

Received 27 November 2002

In revised form 21 February 2003

Accepted 9 March 2003

\section{REFERENCES}

1 Onari K, Spatz H. Anatomische Beitrzezur Lehre von der Pickchen umschriebenen Grosshirnrinden-atrophie ("Picksche Krankheit"). Z Ges Neurol Psychiatr 1926;101:470-511

2 Pick A. Ueber die Beziehungen der senilen Hirnatrophie zur Aphasie. Prag med Wochennschr 1892;17:165-67.

3 Lund and Manchester Research Groups. Clinical and neuropathological criteria for frontotemporal dementia [review]. J Neurol Neurosurg Psychiatry 1994;57:416-18

4 Neary D, Snowden JS, Gustafson L, et al. Frontotemporal lobar degeneration: a consensus on clinical diagnostic criteria. Neurology 1998:51:1546-54.

5 Iseki E, Li F, Odawara T, et al. Ubiquitin-immunohistochemical investigation of atypical Pick's disease without Pick bodies. J Neurol Sci 1998;159: 194-201.

6 Odawara T, Iseki E, Kanai A, et al. Clinicopathological study of two subtypes of Pick's disease in Japan. Dement Geriatr Cogn Disord 2003; 15: 19-25.

7 Ikeda K, Akiyama H, Arai T, et al. Morphometrical reappraisal of motor neuron system of Pick's disease and amyotrophic lateral sclerosis with dementia. Acta Neuropathol 2002;104:21-8.

8 Perry EK, Smith CJ, Court JA, et al. Cholinergic nicotinic and muscarinic receptors in dementia of Alzheimer, Parkinson and Lewy body types. J Neural Transm Park Dis Dement Sect 1990;2:149-58.

9 Perry EK, Haroutunian V, Davis KL, et al. Neocortical cholinergic activities differentiate Lewy body dementia from classical Alzheimer's disease. Neuroreport 1994;5:747-9.

10 Shiozaki K, Iseki E, Uchiyama $\mathrm{H}$, et al. Alterations of muscarinic acetylcholine receptor subtypes in diffuse Lewy body disease: relation to Alzheimer's disease. J Neurol Neurosurg Psychiatry 1999;67:209-13.

11 Wood PL, Etienne P, Lal S, et al. A post-mortem comparison of the cortical cholinergic system in Alzheimer's disease and Pick's disease. J Neurol Sci 1983;62:211-17.

12 Hansen LA, Deteresa R, Tobias $\mathrm{H}$, et al. Neocortical morphometry and cholinergic neurochemistry in Pick's disease. Am J Pathol 1988;131:507-18

13 Francis PT, Holmes C, Webster M-T, et al. Preliminary neurochemical findings in non-Alzheimer dementia due to lobar atrophy. Dementia $1993 ; 4: 172-7$.

14 Proctor AW, Qurne M, Francis PT. Neurochemical features of frontotemporal dementia. Dement Geriatr Cogn Disord 1999;10:80-4

15 Ikeda K. Neuropathological discrepancy between Japanese Pick's disease without Pick bodies and frontal lobe degeneration type of frontotemporal dementia proposed by Lund and Manchester Group. Neuropathology 2000;20:76-82.

16 Flynn DD, Ferrari DG, Mash DC, et al. Differential regulation molecular subtypes of muscarinic receptors in Alzheimer's disease. J Neurochem 1995;64:1888-91.

17 Vilaro MT, Wiederhold KH, Palacios JM, et al. Muscarinic M2 receptor mRNA expression and receptor binding in cholinergic and non-cholinergic cells in the rat brain: a correlative study using in situ hybridization histochemistry and receptor autoradiography. Neuroscience 1992:47:367-93.

18 Mizukami K, Kosaka K. Neuropathological study on the nucleus basalis of Meynert in Pick's disease. Acta Neuropathol 1989;78:52-6. 